Article

\title{
Agrobacterium-Mediated Transformation of Chrysanthemum with Artemisinin Biosynthesis Pathway Genes
}

\author{
Aleksey Firsov ${ }^{1, *}$, Tatiana Mitiouchkina ${ }^{1}$, Lyubov Shaloiko ${ }^{1}$, Alexander Pushin ${ }^{1}$, \\ Alexander Vainstein ${ }^{2}$ and Sergey Dolgov ${ }^{1}$ \\ 1 Branch of the Shemyakin-Ovchinnikov Institute of Bioorganic Chemistry of the RAS, Moscow Region, \\ 142290 Pushchino, Russia; tatiana@planta.bio (T.M.); shaloiko@yandex.ru (L.S.); aspushin@gmail.com (A.P.); \\ dolgov@bibch.ru (S.D.) \\ 2 The Hebrew University of Jerusalem, Robert H. Smith Faculty of Agriculture, Food and Environment, \\ POB 12, Rehovot 76100, Israel; alexander.vainstein@mail.huji.ac.il \\ * Correspondence: aleksey_firsov@mail.ru
}

Received: 25 March 2020; Accepted: 17 April 2020; Published: 21 April 2020

\begin{abstract}
Artemisinin-based drugs are the most effective medicine for the malaria treatment. To date, the main method of artemisinin production is its extraction from wormwood plants Artemisia annua L. Due to the limitation of this source, considerable efforts are now directed to the development of methods for artemisinin production using heterologous expression systems. Artemisinin is a sesquiterpene lactone, synthesized through the cyclization of farnesyl diphosphate involved in other sesquiterpene biosynthetic systems. Chrysanthemum species as well as $A$. annua, belong to Asteraceae family, and had been characterized by containing highly content of sesquiterpenes and their precursors. This makes chrysanthemum a promising target for the production of artemisinin in heterologous host plants. Chrysanthemum (C. morifolium Ramat.) was transformed by Agrobacterium tumefaciens carrying with the binary vectors p1240 and p1250, bearing artemisinin biosynthesis genes coding: amorpha-4,11-diene synthase, artemisinic aldehyde $\Delta 11$ (13) reductase, amorpha-4,11-diene monooxygenase (p1240 was targeted to the mitochondria and p1250 was targeted to the cytosol), cytochrome P450 reductase from A. annua, as well as yeast truncated 3-hydroxy-3-methylglutarylcoenzyme A reductase. This study obtained 8 kanamycin-resistant lines after transformation with the p1240 and 2 lines from p1250. All target genes were detected in 2 and 1 transgenic lines of the 2 vectors. The transformation frequency of all target genes were $0.33 \%$ and $0.17 \%$ for p1240 and p1250, relative to the total transformed explant numbers. RT-PCR analysis revealed the transcription of all transferred genes in two lines obtained after transformation with the p1240 vector, confirming the possibility of transferring genetic modules encoding entire biochemical pathways into the chrysanthemum genome. This holds promise for the development of a chrysanthemum-based expression system to produce non-protein substances, such as artemisinin.
\end{abstract}

Keywords: transgenic plants; chrysanthemum; artemisinin; metabolic engineering

\section{Introduction}

Currently, the most effective drugs for the treatment of malaria are drugs combined with artemisinin, the use of such drugs is recommended by WHO resolution (WHA60.18, May 2007). Additionally, artemisinin and its derivatives are also effective to a number of viruses, many lines of human tumor cells, some tropical parasitic diseases and a number of livestock diseases [1]. Artemisinin is a terpenic lactone found in wormwood plant (Artemisia annua L.). The content of artemisinin in A. annua is usually in the range of $0.01-0.8 \%$ of dry weight, which is not enough to meet the global 
demand for artemisinin-containing drugs [2,3]. Improvement of cultivation techniques and breeding of A. annua has not led to a significant improvement in the yield of artemisinin [4,5]. In this connection, the possibility of producing artemisinin by methods alternative to extraction from natural sources is currently being actively studied. The biochemical pathway of artemisinin synthesis has now been comprehensively studied [6,7] and this allowed advancing research on artemisinin production in heterologous systems.

For example, Farhi et al. (2011) transferred a genetic module from 5 genes of artemisinin synthesis pathway by agrobacterium-mediated method to the tobacco plants [8]. The accumulation of artemisinin in tobacco was $0.0068 \mathrm{mg} / \mathrm{g}$ dry leaf weight (DW). In stable transformed moss of Physcomitrella patens artemisinin accumulation reached to $0.2 \mathrm{mg} / \mathrm{g}$ DW [7]. In experiments on transient expression of artemisinin biosynthesis genes in Nicotiana benthamiana plants, artemisinin accumulated at a level of $0.003 \mathrm{mg} / \mathrm{g}$ DW [9]. A highest level $(0.8 \mathrm{mg} / \mathrm{g}$ DW of leaf tissues) of artemisinin accumulation was observed in double transformed tobacco plants, the artemisinin pathway genes were transferred into the genome and the mevalonate pathway genes (MVA pathway) were transferred in the plastome [10]. These studies confirmed the fundamental possibility of artemisinin production in heterologous plants.

Artemisinin is synthesized from two isoprenoid precursors, isopentenyl diphosphate (IPP) and dimethylallyl diphosphate (DMAPP). IPP and DMAPP are condensed by farnesyl diphosphate synthase (FPPS/FPS) into farnesyl diphosphate (FPP, farnesyl pyrophosphate), the C15 sesquiterpenoid precursor [6,11]. FPP is converted by amorpha-4,11-diene synthase (ADS) to amorpha-4,11-diene (AD) and further into artemisinin (Figure 1). The limited pool of IPP and DMAPP will restrict FPP production and consequently that of artemisinin. This assumption is supported by a study of Malhotra et al. (2016) [10], when the transfer of the mevalonate pathway genes into the plastome of tobacco led to a strong increase of artemisinin accumulation. In this study, a direct correlation was observed between the synthesis of IPP and DMAPP as well the artemisinin production.

An obvious way to increase artemisinin production in heterologous systems is to select a plant-host with a naturally high content of terpenoids and their precursors. Many such plants are known, especially among crop plant belonging to flavor herbs or medicines, such as chrysanthemum [12-14]. The high content of various terpenoids is characteristic of the widespread Chrysanthemum morifolium Ramat, which is one of the leading ornamental crops, second to roses, cultivated almost everywhere $[15,16]$. Noteworthy, the methods of biotechnology and genetic engineering of chrysanthemum have already been developed in detail. The cultivation technologies of chrysanthemum also have been elaborate both for field and for greenhouses conditions [17]. To date, whole-genome sequencing of the diploid species of C. seticuspe and a transcriptome analysis of C. morifolium have been carried out [18,19]. The data obtained in these studies can promote a more in-depth understanding of biochemical processes in chrysanthemum, especially in metabolic engineering. Thus, chrysanthemum may be a promising platform for artemisinin production in planta as a heterologous host.

The aim of this work was to explore the feasibility of agrobacterium-mediated transformation of chrysanthemum with artemisinin biosynthesis genes. Here, we report the successful transformation of chrysanthemum with five genes of artemisinin biosynthesis pathway, i.e., genes responsible for production of dihydroartemisinic acid and mevalonate and show their transcription in the transgenic plants. 


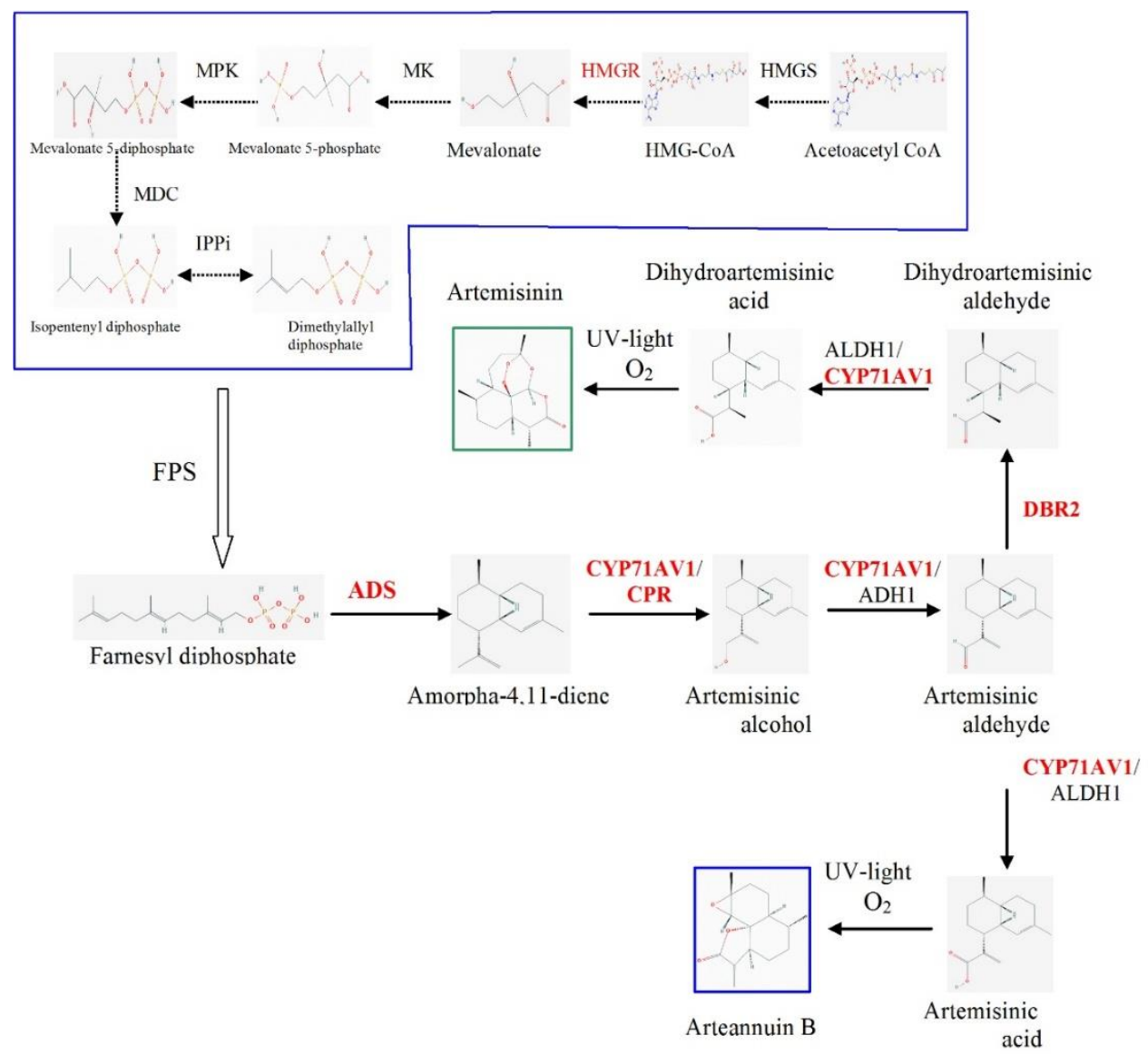

Figure 1. Biosynthetic pathway of artemisinin. The mevalonic acid (MVA) pathway is shown in a blue frame. Artemisinin pathway: ADS: amorpha-4,11-diene synthase; CYP71AV1: amorpha-4,11-diene monooxygenase; CPR: cytochrome P450 reductase; ADH1: alcohol dehydrogenase; DBR2: aldehyde $\triangle 11(13)$ reductase; ALDH1: aldehyde dehydrogenase. MVA pathway: HMGS: 3-hydroxy-3-methylglutaryl-CoA synthase; HMGR: 3-hydroxy-3-methylglutaryl-CoA reductase; MK: mevalonate kinase; MPK: mevalonate 5-phosphate kinase; MDC: mevalonate 5-diphosphate decarboxylase; IPPi: isopentenyl diphosphate isomerase; FPS, farnesyl diphosphate synthase. Artemisinin biosynthesis genes cloned in p1240 and p1250 vectors are shown in red.

\section{Results and Discussion}

\subsection{Agrobacterium-Mediated Transformation of Chrysanthemum Plants with $p 1240$ and $p 1250$ Vectors}

Two variants of the vectors were used in our study-with localization of amorpha-4,11-diene synthase in the cell cytoplasm (p1250)—or in mitochondria (p1240). It was previously reported that switching subcellular localization of introduced ADS to mitochondria leads to a strong increasing of amorpha-4,11-diene accumulation, the artemisinin precursor [20-22]. Studying the influence of artemisinin biosynthesis enzymes localization in various cell compartments on its accumulation is important for the obtaining of plants producing recombinant artemisinin. Vectors p1240 and p1250 were identical and differed only in the presence of a mitochondrial targeting signal sequence in the ADS gene (vector p1240) or its absence (vector p1250) (Figure 2).

Chrysanthemum leaf explants were transformed with the p1240 and p1250 vectors. The mass induction of kanamycin-resistant calli was observed after three weeks of explants cultivation on a regeneration medium with 35.0-mg/L kanamycin (Figure 3A). The calli vigorously grew on a kanamycin-containing medium. The shoot regeneration began after two months of cultivation; the adventitious shoots arose from calli (Figure 3B). On average, 1-2 adventive shoots were formed per one regenerating explant. The green and normally growing regenerants reached $7-10 \mathrm{~mm}$ were cut off 
from the explants and further cultivated on a multiplication medium containing 35-mg/L kanamycin for further selection and proliferation. At this stage, about $80 \%$ of the regenerants turned chlorotic and died, the rest grew and multiplied normally (Figure 3C). When the shoots of kanamycin-resistant shoots reached a height of $3-4 \mathrm{~cm}$, they were planted in the rooting medium containing $35.0-\mathrm{mg} / \mathrm{L}$ kanamycin. After 3 weeks cultivation, the rooted plants with no signs of toxic effect from kanamycin were adapted to the conditions in vivo and then cultivated in a greenhouse (Figure 3D).

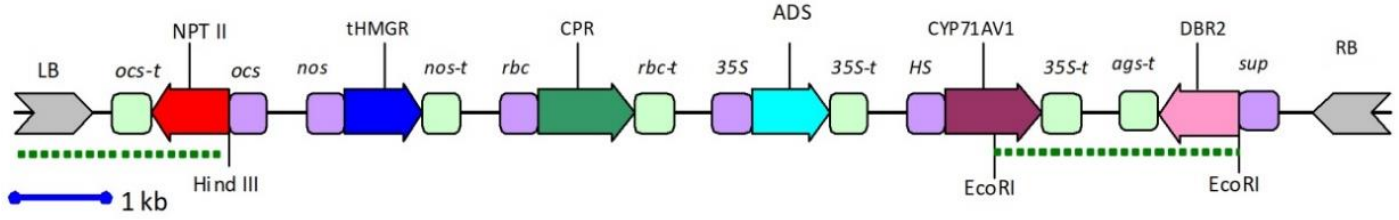

Figure 2. Schematic depiction of the expression cassettes of vectors p1240 and p1250 to artemisinin production in chrysanthemum. tHMGR: 3-hydroxy-3-methylglutaryl-coenzyme A reductase, CPR: cytochrome P450 reductase, ADS (mtADS): amorpha-4,11-diene synthase, CYP71AV1: amorpha-4,11-diene monooxygenase, DBR2: artemisinic aldehyde $\Delta 11$ (13) reductase. Purple boxes indicate promoters, pale green boxes: terminators; ocs: octopine synthase; nos: nopaline synthase; rbc: rubisco; 35S: cauliflower mosaic virus (CaMV) 35S; HS: hps18.1 promoter; sup: superpromoter; ags: agrocinopine synthase. LB and RB: left and right T-DNA borders, respectively. EcoRI and Hind III: sites used for Southern blot analysis. Dotted lines shows probe-specific parts of T-DNA.

A

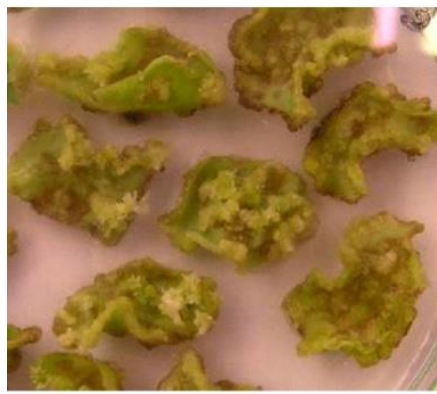

$\mathrm{D}$

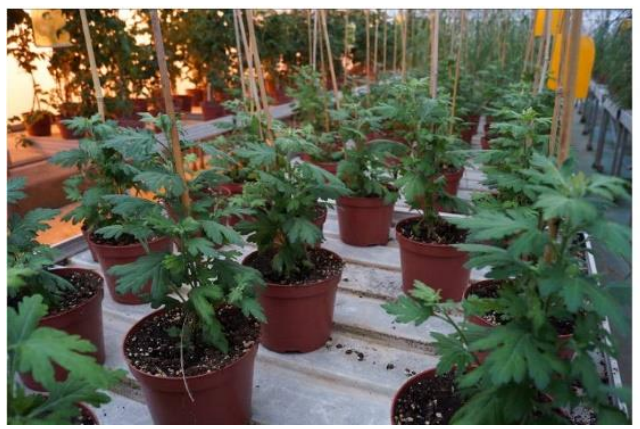

B

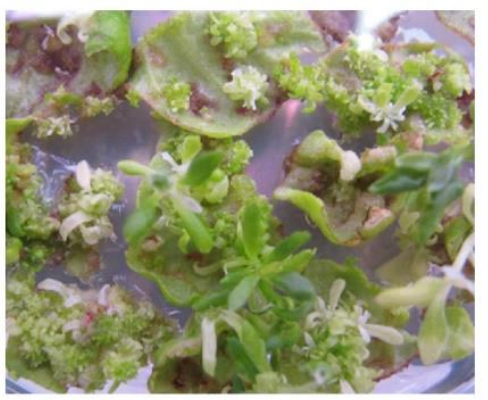

C

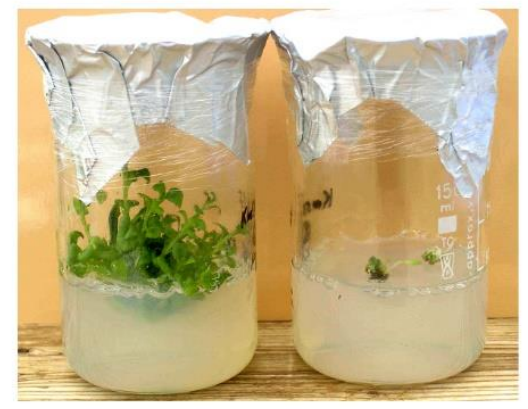

E

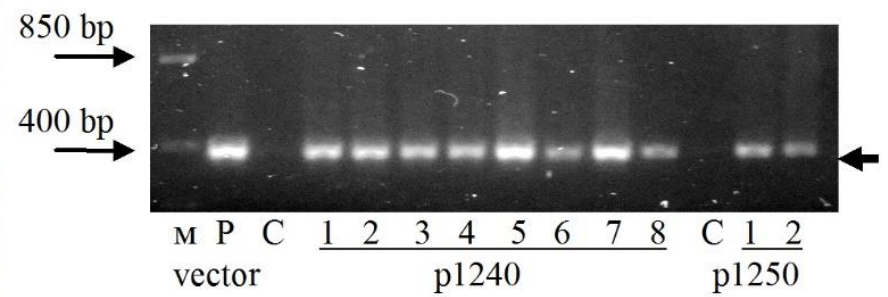

Figure 3. Agrobacterium-mediated transformation of chrysanthemum. (A) Callus induction on the leaf explants. (B) Adventive shoots regeneration on the medium with $35-\mathrm{mg} / \mathrm{L}$ kanamycin. (C) Chrysanthemum transformants on the multiplication medium with kanamycin: on the left is a kanamycin-resistant plant and on the right is sensitive to it. (D) Transgenic chrysanthemum plants in a greenhouse. (E) PCR analysis of NPT II gene in kanamycin-resistant chrysanthemum plants and the expected length of the amplified fragment was $381 \mathrm{bp}$ (arrow indicated). C: nontransformed control plant; P: DNA of p1240. Numbers denote independent chrysanthemum lines. M: molecular size marker. 
At this stage, the kanamycin-resistant chrysanthemum plants were analyzed using PCR to determine the presence of the NPT II gene. A DNA fragment of the expected size was amplified from the DNA samples of all studied kanamycin-resistant lines (Figure 3E). In DNA samples from non-transformed plants, amplification of the NPT II fragment was not observed.

The transgenic chrysanthemum did not differ morphologically from the non-transformed counterparts. The development and growth rate of these plants in the greenhouse did not differ from the corresponding characteristics of the non-transformed plants. In total, ten independent transgenic lines of chrysanthemum were obtained, 8 lines of 1240 and 2 of 1250.

\subsection{Analysis of the Integration of Artemisinin Biosynthetic Pathway Genes in Transformed Plants}

The presence of artemisinin biosynthetic pathway genes in the transgenic chrysanthemum plants were studied using PCR with primers specific to the DBR2, ADS, mtADS, CYP71AV1, tHMGR and CPR genes, respectively. All the target genes were detected in two (1240/1 and 1240/2) out of eight lines, obtained after transformation with vector p1240, and none of these genes were detected in the rest six transgenic lines (Figure 4). All five artemisinin biosynthesis genes were detected in line 1250/1 and the CYP71AV1 gene was not revealed in 1250/2. In DNA samples from non-transformed plants, the fragments of the expected length were not amplified. Taken all together, we obtained 3 lines of chrysanthemum plants transformed with all five target genes.

In addition to PCR products with expected sizes, at using of gene-specific primers for the DBR2, ADS, CYP71AV1 and CPR genes the larger fragments were observed in all DNA samples, including those from non-transformed plants (Figure 4). We hypothesized that this is the result of amplification of chrysanthemum genome sequences, resembling the corresponding sequences from A. annua. Genome sequence analysis of the model species of Chrysanthemum seticuspe confirms the presence of such sequences in chrysanthemum [19]. When used primer pairs in which the forward primer was annealed to the heterologous promoter sequence, the target DBR2 and ADS genes were clearly detectable. Presence of the CPR gene was confirmed using a primer pair amplifying its full-length sequence. The tHMGR gene from yeast was amplified only in transgenic plants.

To further confirm the transgenic origin of the obtained lines, Southern blot analysis (SB) was performed and results matched the results of PCR. Genomic DNA were digested with Hind III followed by SB hybridization, which is expected to yield a DNA fragment longer than $1.7 \mathrm{~kb}$ in length in transgenic plants allowing to estimate the copy number of the T-DNA insertions. Southern blot analysis confirmed the presence of NPT II in the genomes in 4 transgenic lines and the lengths of the detected fragments corresponded with expected (Figure 5A). The NPT II gene in lines 1240/1, 1240/2 and 1250/1 was presented in two copies, in line 1250/2 was single copy.

In the case of restriction with EcoRI, a $2.4 \mathrm{~kb}$ fragment that includes the T-DNA sequence between the EcoRI sites of the DBR2 and CYP71AV1 genes should be revealed (Figure 2). Hybridization of the digested genomic DNA using a probe to DBR2 showed the presence of expected length fragments in the lines 1240/1, 1240/2 and 1250/1 (Figure 5B). In the lines 1240/1 and 1250/1, shorter fragments with a length of 1.2-1.8 kb were also detected. In the 1250/2 line, the fragment was detected corresponding to a size of $1.3 \mathrm{~kb}$, i.e., significantly lower than expected. This may be due to a deletion of the CYP71AV1 gene sequence, which is consistent with the PCR analysis of this line.

$\mathrm{SB}$ analysis results confirmed the integration of vector T-DNA into the genome of studied lines. In lines 1240/1, 1240/2 and 1250/1, the T-DNA insertion is presented in two copies and in line 1250/2 is in one copy that are typical for agrobacterium-mediated transformation. Hybridization with a probe to the DBR2 gene showed the presence of a fragment of the expected length in the lines 1240/1, 1240/2 and $1250 / 1$. In the 1240/2 line, the target fragment was detected as a single band, which indicates the correct transfer of two copies of T-DNA. In lines 1240/1 and 1250/1, SB revealed the presence of additional bands with a molecular weight lower than expected, which point to the incomplete transfer of one of T-DNA copies. The SB analysis also confirmed the PCR results of incomplete T-DNA in the 1250/2 line. 


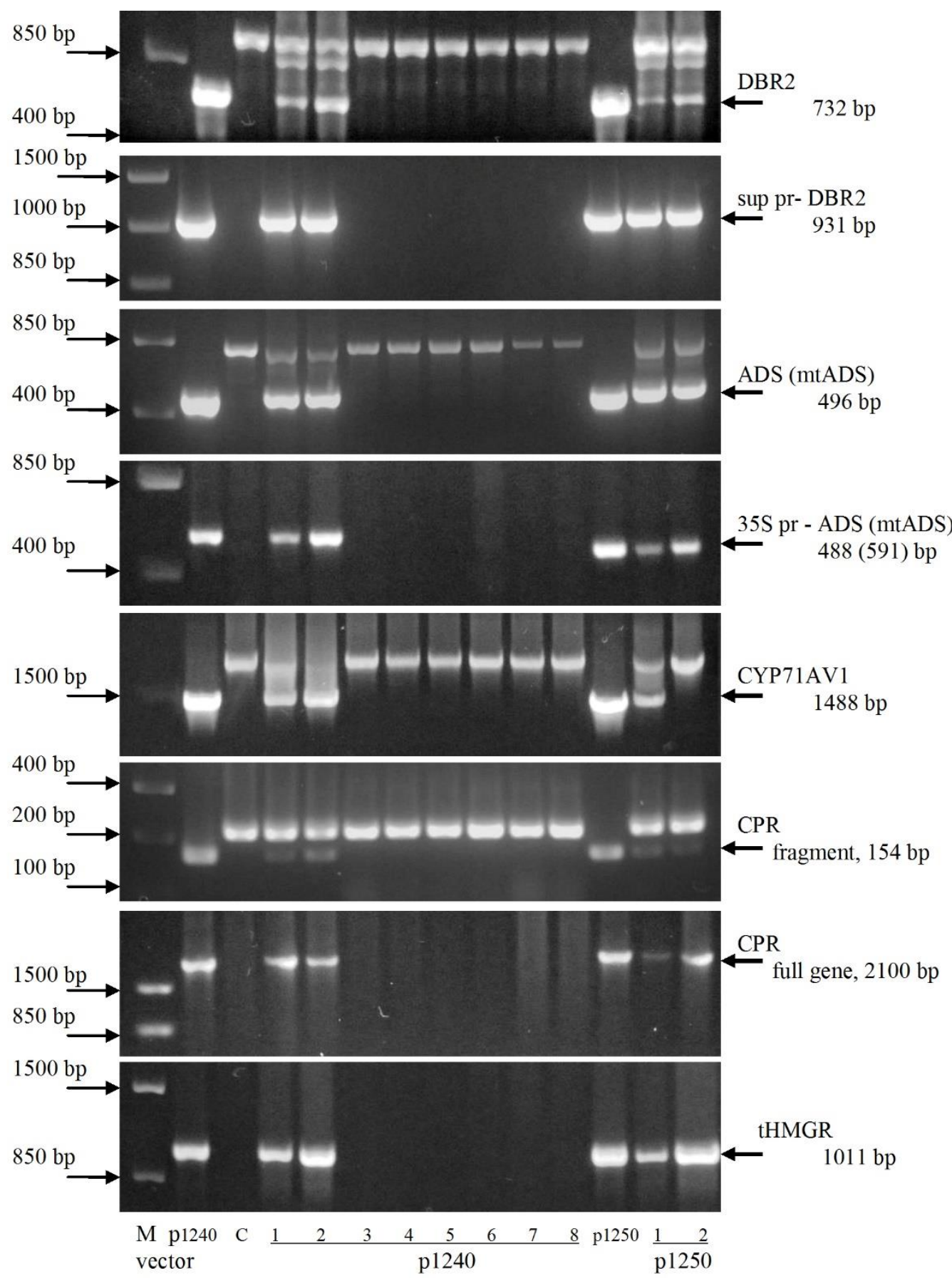

Figure 4. PCR analysis of transgenic chrysanthemum lines. DBR2: artemisinic aldehyde $\Delta 11(13)$ reductase; CYP71AV1: amorpha-4,11-diene monooxygenase; ADS (mtADS): amorpha-4,11-diene synthase; CPR: cytochrome P450 reductase; tHMGR: 3-hydroxy-3-methylglutaryl-coenzyme A reductase. C: nontransformed plant, negative control; p1240 and p1250: DNA of plasmid p1240 and p1250, respectively, positive control. Numbers denote independent transgenic lines. The arrow marks the target fragments. The expected amplified fragments lengths are indicated by bp. M: molecular size marker. 

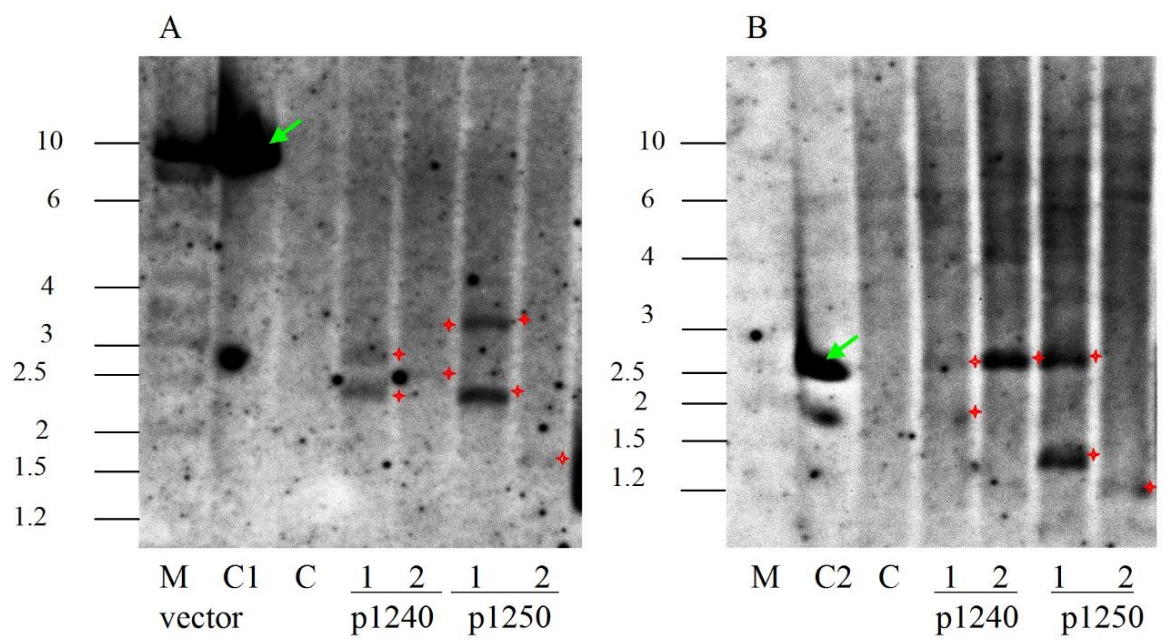

Figure 5. Southern blot analysis of transgenic chrysanthemum lines. (A) The hybridization result of probe to NPT II gene followed by digesting the DNA with Hind III; (B) The hybridization result of probe to DBR2 gene followed by digesting the DNA with EcoRI. C1: DNA of plasmid p1240, positive control; C2: DNA of plasmid p1250, positive control; C: nontransformed plant, negative control. Numbers denote independent transgenic lines. M: molecular size marker, kb. Stars mark positive signals after hybridization with probe. The arrow indicates the signals after hybridization with positive controls.

\subsection{RT-PCR Analysis of Artemisinin Biosynthesis Genes Expression}

The expression of artemisinin biosynthesis genes at the transcription level in lines 1240/1, 1240/2, 1250/1 and 1250/2 were studied using RT-PCR (Figure 6). Fragments corresponding to cDNA sequence of ADS gene were amplified in all transgenic lines. Amplification of tHMGR cDNA fragments was also observed in transgenic plants except for line 1250/1; in this line, the corresponding fragment was not amplified. This is probably due to the gene sequence damage during the T-DNA transfer into the plant genome. In non-transformed plants, amplification of ADS and tHMGR cDNA was not observed.

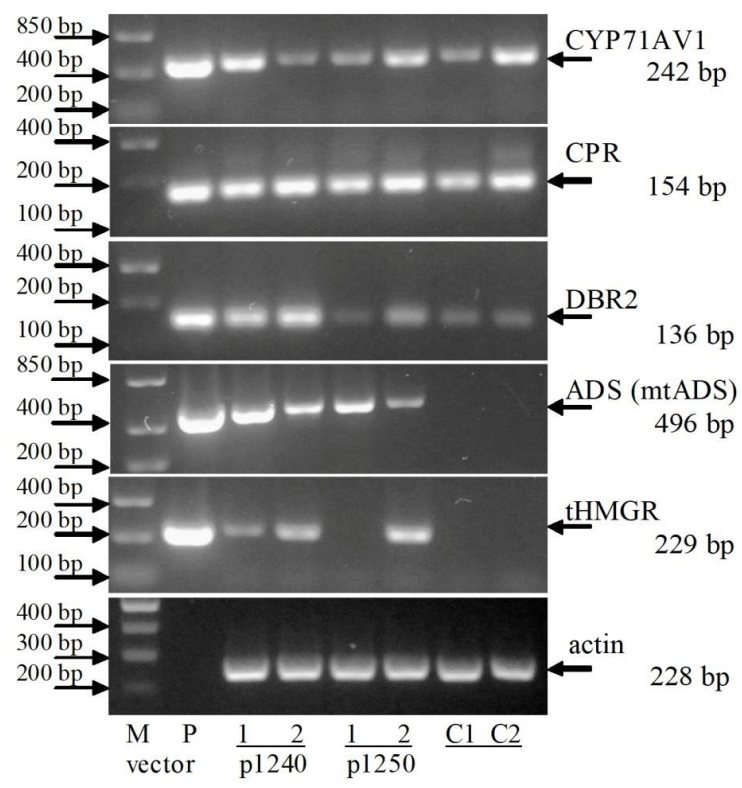

Figure 6. RT-PCR analysis of chrysanthemum lines. $\mathrm{C} 1$ and $\mathrm{C} 2$ : nontransformed plants, negative controls; P: DNA of plasmid p1240, positive control. Actin: actin of C. morifolium, RT-PCR control. Numbers denote independent transgenic lines. M: molecular size marker. The expected length of the amplified fragment are indicated by bp. 
Using primer pairs corresponding to CYP71AV1, CPR and DBR2, the cDNA fragments were amplified both in transgenic lines and in samples obtained from non-transformed plants. This is consistent with the results of PCR, when using gene-specific primers amplification of the corresponding fragments with genome DNA was observed in samples obtained from both transgenic and control plants (Figure 4). The overall results of chrysanthemum transformation experiments and RT-PCR analysis of target genes transcription are shown in Table 1.

Table 1. Summarized results of chrysanthemum transformation with vectors p1240 and p1250.

\begin{tabular}{ccc}
\hline \multirow{2}{*}{ Stages of chrysanthemum transformation and analysis } & \multicolumn{2}{c}{ Vector } \\
\cline { 2 - 3 } Transformed explants & 1240 & 1250 \\
\hline The explants with regenerants & 600 & 600 \\
\hline Adventive shoots per one regenerating explant & 20 & 18 \\
\hline Planted regenerants on the proliferation medium with kanamycin & 1.2 & 1.3 \\
\hline The regenerants died on the proliferation medium with kanamycin & 16 & 23 \\
\hline Kanamycin-resistant lines & 8 & 21 \\
\hline NPT II -positive lines & 8 & 2 \\
\hline Lines with all target genes & 2 & 2 \\
\hline Transcription of all target genes & 2 & 1 \\
\hline
\end{tabular}

\subsection{Artemisinin Assay in the Transgenic Plants}

A TLC analysis was performed to preliminary evaluate the artemisinin accumulation. The lines $1240 / 1$ and 1240/2 were chosen for the study, in these lines the transcription of all target genes was confirmed. Line 1250/1, in which the transcription of the tHMGR gene was not detected; 1250/2, into which the CYP71AV1 gene was not transferred, were not analyzed.

The results of TLC analysis are shown in Figure 7. In the studied lines, after development with anisaldehyde, a band was detected that was absent in non-transformed plants. The band had a pink color, typical of artemisinin. In addition, in this region of the chromatogram, a clearly visible unidentified blue band was observed, which was absent in untransformed plants. The band corresponding to artemisinic acid was not detected in the samples. Thus, the results of TLC analysis indicate a modification of plant metabolism after their transformation by artemisinin pathway genes.

As a result of the experiments, we obtained eight chrysanthemum lines after transformation with the p1240 vector and two lines after transformation with the p1250 vector. Based on the number of kanamycin-resistant lines, the transformation frequency was $1.3 \%$ and $0.3 \%$ of transformed explants. Of the eight transgenic lines obtained after transformation with the p1240, all target genes were detected in two lines and only one line containing all five target genes was obtained after transformation with the p1250. Thus, the frequency of chrysanthemum transformation of all target genes were between $0.33 \%$ and $0.17 \%$. In most studies, the frequency of chrysanthemum transformation of varies in the range of $1-8 \%$ of transformed explants number [23-25]. The values are not high in this study. Since previous studies were transferred only 2-3 genes to the chrysanthemum genome, the chrysanthemum transformation by a large number of genes are unknown to us.

Currently, studies in the field of multiple gene transfer are carried out using model plants and easily transformed crops. For instance, 10 different genes as part of 28.5-kb T-DNA were possibly transferred into the Arabidopsis thaliana genome [26], 8 genes important for symbiosis with rhizobia (T-DNA size of $74 \mathrm{~kb}$ ) were able to be transferred into Medicago truncatula [27], seven carotenoid biosynthesis genes in a single $17 \mathrm{~kb}$ T-DNA were able to be transferred into Brassica napus [28] and 32-kb T-DNA with ten insect resistance genes were successfully introduced into the elite line of maize 
and their expression in plants was shown [29]. This study was the first that introduced six genes simultaneously into the chrysanthemum genome with a size of about $14.0 \mathrm{~kb}$ T-DNA.

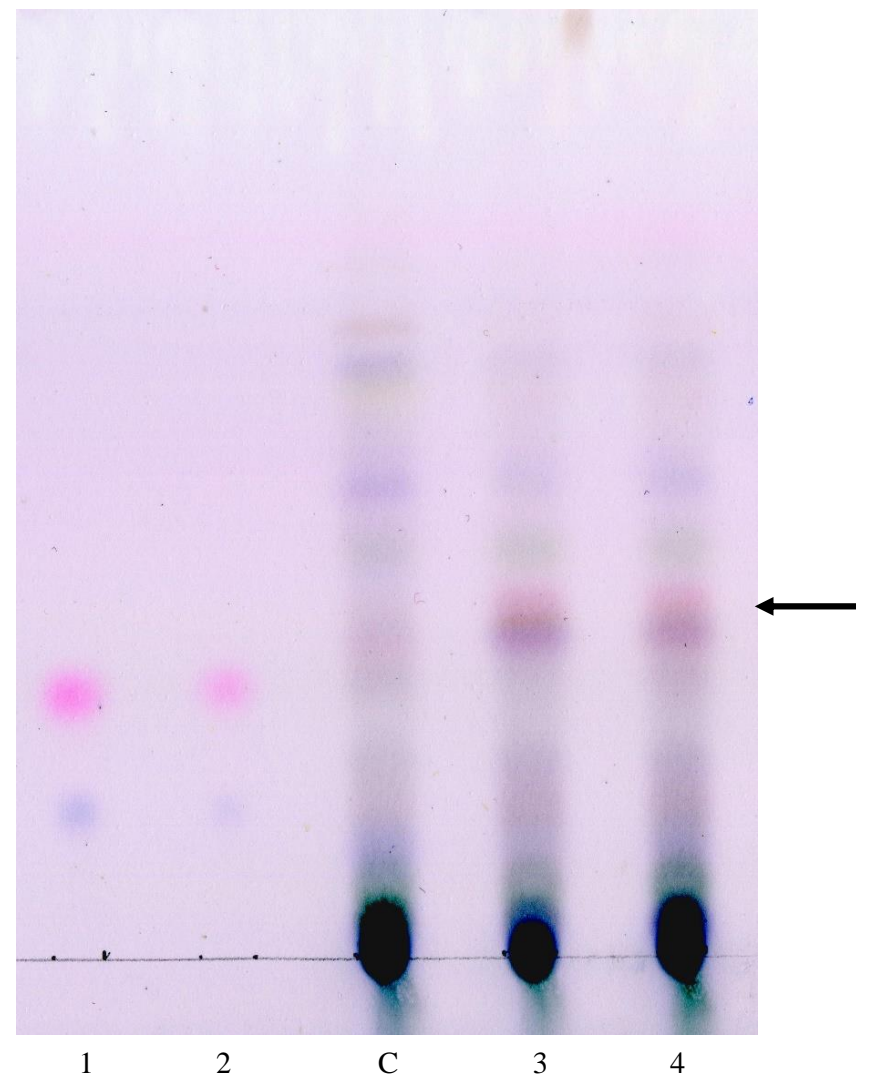

Figure 7. TLC analysis of artemisinin accumulation in transgenic chrysanthemum plants. Lanes 1 and 2: mixed standards of artemisinin and artemisinic acid (125 ng of each, lane 1; $62.5 \mathrm{ng}$ of each, lane 2); C: non-transformed plant; 3: line 1240/1; 4: line 1240/2.

It should be noted that even small differences in the nucleotide sequence of T-DNA had a significant impact on the efficiency of its transfer during transformation. During transformation with the p1240 vector, only in two lines did the transfer of target genes take place; in the other 6 lines, none of them was detected. At the same time, during transformation with the p1250 vector, which differed from p1240 only in the absence of a signal peptide sequence in the ADS gene, the transfer of target genes was observed in both lines obtained.

RT-PCR analysis confirmed the transcription of heterologous ADS and tHMGR genes in transgenic plants. At the same time, using gene-specific primers for CYP71AV1, CPR and DBR2, amplification of cDNA fragments was observed both in transgenic lines and in non-transformed plants. TLC analysis revealed bands in the studied transgenic lines that were absent in the control plants, including those with a pink color typical of artemisinin. These data indicate a modification in the metabolism of transgenic plants under the influence of artemisinin pathway genes. Evaluation the recombinant artemisinin accumulation in the transgenic plants and a more thorough analysis of various genes influence on the artemisinin biosynthesis will be the next step of our study.

For metabolic engineering, it may be more applicable to transfer the biosynthesis module genes into plants not as one large T-DNA, but as a part of several smaller T-DNAs. Such a transfer can be carried out, in particular, by the method of co-transformation, when the explants are transformed simultaneously with several vectors. Other approach is the transformation of previously obtained and characterized lines carrying biosynthetic module genes (or part of them) with vectors with others studied genes (double transformation). It is also possible to obtaining a set of transgenic lines, each of 
which carries a part of the genetic module, with the subsequent crossing between them. These methods allow greater research flexibility by opportunities of combining various biosynthesis genes of the target product or its precursors. In addition, the last two approaches allow the use in studies the lines with the maximum expression of heterologous genes, thereby minimizing T-DNA position effect. The effectiveness of these approaches has been repeatedly confirmed; they will be used in our further research.

Thus, the data obtained in this study show the possibility of transferring gene groups encoding whole biochemical modules into the chrysanthemum genome. This opens up new routes for the development of chrysanthemum plant-based expression systems for the production of non-protein substances, including artemisinin.

\section{Materials and Methods}

\subsection{The Plant Transformation Vectors}

The chrysanthemum plants were transformed with p1240 and p1250 vectors. The vectors were constructed by cloning the genes of the artemisinin biosynthesis pathway into the pRCS16F vector using intermediate vectors of the pSAT series [8,30]. The following genes of Artemisia. annua were cloned into the pRCS16F vector: amorpha-4,11-diene synthase (ADS), artemisinic aldehyde $\Delta 11$ (13) reductase (DBR2) and amorpha-4,11-diene monooxygenase (CYP71AV1). CYP71AV1 catalyzes amorpha-4,11-diene to form artemisinic aldehyde via artemisinic alcohol as well as conversion dihydroartemisinic aldehyde into dihydroartemisinic acid (Figure 1). Cytochrome P450 reductase (CPR) from A. annua was cloned into vector to prevent the accumulation of inactive oxidized P450. The vectors also contained a truncated and deregulated 3-hydroxy-3-methylglutarylcoenzyme A reductase (tHMGR) from yeast to increase the supply of precursor from the mevalonate (MVA) pathway for artemisinin production.

The amorpha-4,11-diene synthase gene was cloned in two variants-without a signal sequence (localization of ADS in the cytosol, vector p1250) and with the signal sequence of cytochrome C oxidase subunit 4 (COX4) from Saccharomyces cerevisiae (mtADS, localization in mitochondria, vector p1240). Thus, the engineered vectors differed from each other solely in the absence (p1250) or presence ( $\mathrm{p} 1240)$ of the COX4 signal peptide nucleotide sequence. The expression cassettes of vectors used in this study are presented in Figure 2.

The plasmids were transferred into Agrobacterium tumefaciens CBE21 and used for transformation. A. tumefaciens was grown overnight in liquid LB medium at $28^{\circ} \mathrm{C}$ and $140 \mathrm{rpm}$. The bacterial cells were washed twice in liquid hormone-free Murashige and Skoog (MS) medium by centrifugation for $5 \mathrm{~min}(4000 \times \mathrm{g})$, and the bacterial pellet was resuspended in this medium to an OD600 of 0.2.

\subsection{Agrobacterial Transformation of Chrysanthemum}

The chrysanthemum plants (Chrysanthemum morifolium Ramat) of the cultivar White Snowdon were used in all experiments. Agrobacterium-mediated transformation and in vitro culture of chrysanthemum plants were performed as described previously [31,32].

Briefly, stock chrysanthemum plant were multiplicated on Murashige and Skoog medium (MS) with 1.0-mg/L 6-benzylaminopurine (BA), 0.1-mg/L indole-3-acetic acid, 0.5-mg/L gibberellic acid, $30 \mathrm{~g} / \mathrm{L}$ sucrose and $0.8 \%$ agar. The shoot of $1.5-2.0 \mathrm{~cm}$ were rooted on the rooting medium (contained two times diluted MS salts, containing the macro- and micro-elements, vitamins MS, $10 \mathrm{~g} / \mathrm{L}$ sucrose and $0.5-\mathrm{mg} / \mathrm{L}$ indolebutyric acid). The explants (the leaf pieces approximately $1 \times 1 \mathrm{~cm}$ ) were cut from three weeks old rooted plants. The leaf explants were incubated in bacterial suspension for $20 \mathrm{~min}$, then placed upside down on regeneration medium (MS medium with 2.0-mg/L BA, 0.5-mg/L naphthaleneacetic acid) without antibiotics. After three days co-cultivation with agrobacterium, the explants were transferred to the regeneration medium (the regeneration medium containing of $500-\mathrm{mg} / \mathrm{L}$ cefotaxim and 35-mg/L kanamycin) and were subcultured every 3 weeks. The regenerants 
reaching 7-10 $\mathrm{mm}$ were cut off from the explants and further cultivated on a multiplication medium with 35.0-mg/L kanamycin and 500-mg/L cefotaxim. When the kanamycin-resistant shoots reached a height of 3 to $4 \mathrm{~cm}$, they were transferred on the rooting medium containing 35.0-mg/L kanamycin and $250-\mathrm{mg} / \mathrm{L}$ cefotaxim. The rooted, vigorously growing kanamycin-resistant plants were than adapted to conditions in vivo and cultivated in a greenhouse.

\subsection{PCR and Southern Blot Analysis}

For PCR and Southern blot analysis, the genomic DNA of chrysanthemum was isolated from kanamycin-resistant and nontransformed control plants grown in greenhouse using the method of Dellaporta et al. [33]. DNA preparations were checked for suitability to PCR amplification using primers to actin of chrysanthemum (Figure S1A).

The plants were pre-tested for the absence of agrobacterial contamination using primers virBF and virBR that amplify the virB gene of $A$. tumefaciens (Table S1). The primer sequences used for detection of artemisinin biosynthesis genes in transgenic plants; the PCR regimes are presented in Table S1 of Supplementary Material.

Chrysanthemum genomic DNA $(70 \mu \mathrm{g})$ was digested overnight at $37^{\circ} \mathrm{C}$ with $100 \mathrm{U}$ Hind III (cuts the T-DNA of p1240 and p1250 between the NPT II coding sequence and its octopine synthase promoter) or EcoRI (cuts out DNA fragment between DBR2 coding sequence and CYP71AV1) (Figure 2). Digestion by Hind III was used to evaluate the copy number of the T-DNA inserts, EcoRI allows to further confirm the integration of right border (RB) part of T-DNA into the plant genome. The DNA of non-transformed chrysanthemum plants digested with Hind III or EcoRI was used as a negative control.

After agarose gel ( $0.8 \%)$ electrophoresis, the digestion products were transferred and immobilized onto Hybond+ membrane (Amersham, USA) following the manufacturer's instructions. The DNA probe was constructed by PCR using plasmid p1240 as the template and primer pairs NPT IIF-NPT IIR and DBR2F-DBR2R to amplify the sequences of NPT II and DBR2 genes. DNA probes (381 bp of NPT II and 732 bp of DBR2) were labeled with alkaline phosphatase using the AlkPhos Direct Labeling Kit (Amersham Bioscience, USA). Prehybridization, hybridization (incubated at $60^{\circ} \mathrm{C}$ overnight) with alkaline phosphatase labeled probe and subsequently washings of the membrane were carried out according to the AlkPhos Direct Labeling Kit protocol. Detection was performed using CDP-Star detection reagent following the manufacturer's directions (Amersham Bioscience).

\subsection{Reverse Transcription-Polymerase Chain Reaction Analysis}

The complete transgenic lines, 1240/1, 1240/2 and 1250/1, were further analyzed by RT-PCR. Non-transformed plants as well as the incomplete T-DNA transformation line, 1250/2, were used as a negative control. The total RNA was isolated from the leaves with the QuantumPrep AquaPure RNA Isolation kit (BioRad, Hercules, CA, USA) according to the manufacturer's recommendations. The obtained RNA was additionally purified from DNA impurities via treatment with DNAse (Thermo Fisher Scientific, Waltham, MA, USA). RNA preparations were checked for the absence of DNA contamination by PCR analyses without reverse transcription step (Figure S1B). Reverse transcription was performed using an M-MuLV RT enzyme (Thermo Fisher Scientific, USA) and oligo d(T) ${ }_{16}$ primer following the manufacturer's directions. The obtained cDNA preparations were analyzed by PCR for the presence of target sequences. The primer sequences and RT-PCR conditions are presented in Table S2 of Supplementary Material.

\subsection{Artemisinin Assay in the Transgenic Plants}

Artemisinin assay was performed using TLC $[34,35]$. Chemicals used were of analytical grade. Anisaldehyde, $n$-hexane, trifluoroacetic acid (TFA), artemisinin, artemisinic acid were from Sigma (USA); glacial acetic acid, $\mathrm{H}_{2} \mathrm{SO}_{4}$ and methanol were from Panreac (USA); RP-18 ${ }_{\mathrm{F} 2545}$ TLC plate-Merck (Germany). Artemisinin and artemisinic acid were used as external standards. The standards were 
dissolved in methanol at of concentration of $1 \mathrm{mg} / \mathrm{mL}$; the working solutions were prepared by diluting the stock solutions with methanol up to 125.0 and $62.25 \mathrm{ng} / \mu \mathrm{L}$.

The leaves of transgenic and control non-transformed plants growing in the greenhouse were collected, dried in a hot air oven at $56^{\circ} \mathrm{C}$ for $18 \mathrm{~h}$ and powdered. The samples (100 mg) were extracted overnight in $n$-hexane $(10 \mathrm{~mL})$, filtered, evaporated under $\mathrm{N}_{2}$ gas flow and dissolved in $1.0 \mathrm{~mL}$ of methanol. On the TLC plates the samples and standard solutions were applied manually, $10 \mu \mathrm{L}$ and $1 \mu \mathrm{L}$ of each, respectively. The linear ascending development was carried out on RP- 18 F254S TLC plate, $20 \times 10 \mathrm{~cm}$ in a twin-trough chamber presaturated with mobile phase $0.2 \%$ TFA in water/ACN (35:65, $v / v)$. The chromatogram was allowed to develop for $35 \mathrm{~min}$ to a height of $8 \mathrm{~cm}$ at room temperature $\left(25 \pm 2{ }^{\circ} \mathrm{C}\right)$ and $50 \% \pm 5 \%$ relative humidity. After the development, TLC plates were dried in a current of air with the help of an air fan. The dried plate was dipped into freshly prepared reagent consisting of glacial acetic acid/concentrated $\mathrm{H}_{2} \mathrm{SO}_{4}$ /anisaldehyde (50:1:0.5, $v / v / v$ ) followed by heating at $110{ }^{\circ} \mathrm{C}$ for $5 \mathrm{~min}$ to visualize the bands of artemisinin. TLC plates were visualized using imaging system Amersham Imager 600RGB (GE, USA). Artemisinin TLC analyses were performed in triplicate.

Supplementary Materials: The following are available online at http://www.mdpi.com/2223-7747/9/4/537/s1, Figure S1: Quality check of DNA and RNA preparations for PCR and RT-PCR analysis, Table S1: PCR regimes and nucleotide sequences of used primers, Table S2: RT-PCR conditions and nucleotide sequences of used primers.

Author Contributions: Conceptualization, A.V.; investigation, A.F., T.M., L.S. and A.P.; project administration, S.D.; supervision, S.D.; writing — original draft, A.F.; writing—review \& editing, A.V. All authors have read and agreed to the published version of the manuscript.

Funding: The work was supported by the Russian Science Foundation, Grant No. 19-14-00190 using the unique scientific facility Fitotron reg. \#2-2.9.

Acknowledgments: We are grateful to A. Blagova for her valuable technical assistance.

Conflicts of Interest: The authors declare no conflict of interest.

\section{References}

1. Ho, W.; Peh, H.; Chan, T.; Wong, W.S. Artemisinins: Pharmacological actions beyond anti-malarial. Pharm. Ther. 2014, 142, 126-139. [CrossRef] [PubMed]

2. Johnston, G. Artemisia annua, Artemisinin, ACTs \& Malaria Control in Africa: Tradition, Science and Public Policy by Dana G. Dalrymple. Q. Rev. Biol. 2013, 88, 257-258.

3. Brisibe, E.A.; Chukwurah, P.N. Production of artemisinin in planta and in microbial systems need not be mutually exclusive. In Artemisia annua-Pharmacology and Biotechnology; Aftab, T., Ferreira, J.F.S., Eds.; Springer: Berlin/Heidelberg, Germany, 2014; pp. 269-292.

4. Kumar, S.; Gupta, S.; Singh, P.; Bajpai, P.; Gupta, M.; Singh, D.; Gupta, A.; Ram, G.; Shasany, A.; Sharma, S. High yields of artemisinin by multi-harvest of Artemisia annua crops. Ind. Crops Prod. 2004, 19, 77-90. [CrossRef]

5. Tu, Y. Metabolic Engineering of Artemisia annua L. In From Artemisia annua L. to Artemisinins. The Discovery and Development of Artemisinins and Antimalarial Agents; Chemical Industry Press: Beijing, China, 2017; Chapter 8; pp. 163-208.

6. Brown, G.D. The biosynthesis of artemisinin (Qinghaosu) and the phytochemistry of Artemisia annua L. (Qinghao). Molecules 2010, 15, 7603-7698. [CrossRef] [PubMed]

7. Ikram, N.K.B.; Beyraghdar Kashkooli, A.; Peramuna, A.V.; van der Krol, A.R.; Bouwmeester, H.; Simonsen, H.T. Stable production of the antimalarial drug artemisinin in the moss Physcomitrella patens. Front. Bioeng. Biotechnol. 2017, 5, 47. [CrossRef] [PubMed]

8. Farhi, M.; Marhevka, E.; Ben-Ari, J.; Algamas-Dimantov, A.; Liang, Z.; Zeevi, V.; Edelbaum, O.; Spitzer-Rimon, B.; Abeliovich, H.; Schwartz, B.; et al. Generation of the potent anti-malarial drug artemisinin in tobacco. Nat Biotechnol. 2011, 29, 1072-1074. [CrossRef] [PubMed]

9. Wang, B.; Kashkooli, A.B.; Sallets, A.; Ting, H.-M.; De Ruijter, N.C.; Olofsson, L.; Brodelius, P.; Pottier, M.; Boutry, M.; Bouwmeester, H.; et al. Transient production of artemisinin in Nicotiana benthamiana is boosted by a specific lipid transfer protein from A. annua. Metab. Eng. 2016, 38, 159-169. [CrossRef] [PubMed] 
10. Malhotra, K.; Subramaniyan, M.; Rawat, K.; Kalamuddin, M.; Qureshi, M.I.; Malhotra, P.; Mohmmed, A.; Cornish, K.; Daniell, H.; Kumar, S. Compartmentalized metabolic engineering for artemisinin biosynthesis and effective malaria treatment by oral delivery of plant. Cells. Mol. Plant 2016, 9, 1464-1477. [CrossRef]

11. Wen, W.; Yu, R. Artemisinin biosynthesis and its regulatory enzymes: Progress and perspective. Pharmacogn. Rev. 2011, 5, 189-194.

12. Bruno, M.; Bancheva, S.; Rosselli., S.; Maggio, A. Sesquiterpenoids in subtribe Centaureinae (Cass.) Dumort (tribe Cardueae, Asteraceae): Distribution, (13)C NMR spectral data and biological properties. Phytochemistry 2013, 95, 19-93.

13. Wang, Y.; Li, X.; Jiang, Q.; Sun, H.; Jiang, J.; Chen, S.; Guan, Z.; Fang, W.; Chen, F. GC-MS Analysis of the Volatile Constituents in the Leaves of 14 Compositae Plants. Molecules 2018, 23, 166. [CrossRef]

14. Xue, G.-M.; Li, X.-Q.; Chen, C.; Chen, K.; Wang, X.-B.; Gu, Y.-C.; Luo, J.-G.; Kong, L.-Y. Highly Oxidized Guaianolide Sesquiterpenoids with Potential Anti-inflammatory Activity from Chrysanthemum indicum. J. Nat. Prod. 2018, 81, 378-386. [CrossRef] [PubMed]

15. Chang, K.M.; Kim, G.H. Volatile aroma constituents of gukhwa (Chrysanthemum morifolium R.). Food Sci Biotechnol. 2013, 22, 659-663. [CrossRef]

16. Yang, L.; Nuerbiye, A.; Cheng, P.; Wang, J.H.; Li, H. Analysis of Floral Volatile Components and Antioxidant Activity of Different Varieties of Chrysanthemum morifolium. Molecules 2017, 22, E1790. [CrossRef] [PubMed]

17. Spaargaren, J.; van Geest, G. Chrysanthemum. In Ornamental Crops; Van Huylenbroeck, J., Ed.; Springer International Publishing: Cham, Switzerland, 2018; pp. 319-348.

18. Won, S.Y.; Kwon, S.-J.; Lee, T.-H.; Jung, J.-A.; Kim, J.S.; Kang, S.-H.; Sohn, S.-H. Comparative transcriptome analysis reveals whole-genome duplications and gene selection patterns in cultivated and wild Chrysanthemum species. Plant Mol. Biol. 2017, 95, 451-461. [CrossRef]

19. Hirakawa, H.; Sumitomo, K.; Hisamatsu, T.; Nagano, S.; Shirasawa, K.; Higuchi, Y.; Kusaba, M.; Koshioka, M.; Nakano, Y.; Yagi, M.; et al. De novo whole-genome assembly in Chrysanthemum seticuspe, a model species of Chrysanthemums, and its application to genetic and gene discovery analysis. Dna Res. 2019, 26, 195-203. [CrossRef]

20. Van Herpen, T.W.J.M.; Cankar, K.; Nogueira, M.; Bosch, D.; Bouwmeester, H.J.; Beekwilder, J. Nicotiana benthamiana as a Production Platform for Artemisinin Precursors. PLoS ONE 2010, 5, e14222. [CrossRef]

21. Farhi, M.; Marhevka, E.; Masci, T.; Marcos, E.; Eyal, Y.; Ovadis, M.; Abeliovich, H.; Vainstein, A. Harnessing yeast subcellular compartments for the production of plant terpenoids. Metab. Eng. 2011, 13, 474-481. [CrossRef]

22. Farhi, M.; Kozin, M.; Duchin, S.; Vainstein, A. Metabolic engineering of plants for artemisinin synthesis. Biotechnol. Genet. Eng. Rev. 2013, 29, 135-148. [CrossRef]

23. Shinoyama, H.; Mitsuhara, I.; Ichikawa, H.; Kato, K.; Mochizuki, A. Transgenic chrysanthemums (Chrysanthemum morifolium Ramat.) carrying both insect and disease resistance. Acta Hortic. 2015, 1087, 485-497. [CrossRef]

24. Naing, A.H.; Ai, T.N.; Jeon, S.M.; Lim, S.H.; Kim, C.K. An efficient protocol for Agrobacterium-mediated genetic transformation of recalcitrant chrysanthemum cultivar Shinma. Acta Physiol. Plant. 2016, 38, 38. [CrossRef]

25. Mitiouchkina, T.; Firsov, A.; Titova, S.; Shulga, O.; Dolgov, S. Efficiency assessment of genetic designs with coat protein in transgene-mediated resistance against Chrysanthemum virus B. Acta Hortic. 2018, 1193, 89-94. [CrossRef]

26. Collier, R.; Thomson, J.G.; Thilmony, R. A versatile and robust Agrobacterium-based gene stacking system generates high-quality transgenic Arabidopsis plants. Plant J. 2018, 95, 573-583. [CrossRef] [PubMed]

27. Untergasser, A.; Bijl, G.J.; Liu, W.; Bisseling, T.; Schaart, J.G.; Geurts, R. One-step Agrobacterium mediated transformation of eight genes essential for rhizobium symbiotic signaling using the novel binary vector system pHUGE. PLoS ONE 2012, 7, e47885. [CrossRef]

28. Fujisawa, M.; Takita, E.; Harada, H.; Sakurai, N.; Suzuki, H.; Ohyama, K.; Shibata, D.; Misawa, N. Pathway engineering of Brassica napus seeds using multiple key enzyme genes involved in ketocarotenoid formation. J. Exp. Bot. 2009, 60, 1319-1332. [CrossRef] [PubMed]

29. Ward, J.; Xu, J.; Lamb, J.; Gilbertson, L. The use of Agrobacterium tumefaciens to deliver ten genes on a single vector to corn. In Proceedings of the 9th International Plant Molecular Biology (IPMB) Congress 2009, Saint Louis, MO, USA, 25-30 October 2009. 
30. Chung, S.M.; Frankman, E.L.; Tzfira, T. A versatile vector system for multiple gene expression in plants. Trends Plant Sci. 2005, 10, 357-361. [CrossRef] [PubMed]

31. Shulga, O.A.; Mitiouchkina, T.Y.; Shchennikova, A.V.; Skryabin, K.G.; Dolgov, S.V. Overexpression of AP1-like genes from Asteraceae induces early-flowering in transgenic Chrysanthemum plants. In Vitro Cell. Dev. Biol. Plant. 2011, 47, 553-560. [CrossRef]

32. Mitiouchkina, T.Y.; Firsov, A.P.; Titova, S.M.; Pushin, A.S.; Shulga, O.A.; Dolgov, S.V. Different approaches to produce transgenic virus B Resistant Chrysanthemum. Agronomy 2018, 8, 28. [CrossRef]

33. Dellaporta, S.L.; Wood, J.; Hicks, J.B. A plant DNA minipreparation version II. Plant Mol. Biol. Report. 1983, 1, 19-21. [CrossRef]

34. Bhandari, P.; Gupta, A.P.; Singh, B.K.; Vijay, K. Simultaneous densitometric determination of artemisinin, artemisinic acid and arteannuin B in Artemisia annua using reversed-phase thin layer chromatography. J. Sep. Sci. 2005, 28, 2288-2292. [CrossRef]

35. Khan, S.; Ali, A.; Ahmad, S.; Abdin, M.Z. Affordable and rapid HPTLC method for the simultaneous analysis of artemisinin and its metabolite artemisinic acid in Artemisia annua L. Biomed. Chromatogr. 2015, 29, 1594-1603. [CrossRef]

(C) 2020 by the authors. Licensee MDPI, Basel, Switzerland. This article is an open access article distributed under the terms and conditions of the Creative Commons Attribution (CC BY) license (http://creativecommons.org/licenses/by/4.0/). 